\title{
GEOREFERENCING AND REPROJECTION ERROR INVESTIGATION ON IMAGE BASED 3D DIGITIZATION AND MAPPING OF HISTORICAL BUILDINGS
}

\author{
C.Altuntas ${ }^{1, *}$ \\ ${ }^{1}$ Konya Technical University, Engineering and Natural Science Faculty, 42075 Selcuklu, Konya, Turkey - caltuntas@ktun.edu.tr
}

Commission II

KEY WORDS: Dense Point Cloud, Reprojection Error, Georeferencing, Accuracy, Sequential Image Orientation

\begin{abstract}
:
Image based dense point cloud creation is easy and low-cost application for three dimensional digitization of small and large scale objects and surfaces. It is especially attractive method for cultural heritage documentation. Reprojection error on conjugate keypoints indicates accuracy of the model and keypoint localisation in this method. In addition, sequential registration of the images from large scale historical buildings creates big cumulative registration error. Thus, accuracy of the model should be increased with the control points or loop close imaging. The registration of point point cloud model into the georeference system is performed using control points. In this study historical Sultan Selim Mosque that was built in sixteen century by Great Architect Sinan was modelled via photogrammetric dense point cloud. The reprojection error and number of keypoints were evaluated for different base/length ratio. In addition, georeferencing accuracy was evaluated with many configuration of control points with loop and without loop closure imaging.
\end{abstract}

\section{INTRODUCTION}

Photogrammetry and terrestrial laser scanning has been used extensively in documentation of cultural heritage for last two decades. Especially time of flight 3D laser scanner has been popular in 3D measurement and modelling of historical structures and objects because for collecting high density $3 \mathrm{D}$ data in a short time. Dense image matching by using structurefrom-motion (SfM) photogrammetry has recently emerged as an alternative and competing technology for creating dense point cloud. SfM is low cost and fast applicable method than laser scanning. However, An elaborate approach is required for SfM to produce comparable results to terrestrial laser scanner (Wilkinson et al., 2016). SfM is computer vision approach for $3 \mathrm{D}$ photogrammetric measurement from stereoscopic images. It creates conjugate image points between overlapping images and estimates positions of the images and calibration parameters automatically. These set of images are exploited for creating dense point cloud model with more conjugate points. Keypoint detection operators (sift, asift, etc.) are detecting the image points and represent them with feature vector in huge dimensional space. The keypoints from overlapping images are matched with similarity of these feature vectors, and correct matched keypoints constitutes sparse point cloud prior to creating dense point cloud. The reprojection errors on these conjugated keypoints have been used to evaluating the accuracy of estimated camera motions and parameters. On the other hand point of view angle is also affect keypoint number and reprojection error. High reprojection error usually indicates poor localization of the image points. Total reprojection error is an indicator for the global accuracy of the SfM solution (Verhoeven et al., 2015), but this error is strictly related to the amount and position of the tie points extracted during the camera orientation (Piermattei, 2016). Barazzetti et al., (2011) demonstrate that, although images can be relatively orientated among them, an automatic, rigorous and precise result can be obtained if the standard rules of photogrammetry are followed. The reprojection error was evaluated for varying point of view and imaging distances in this study.

The sequential orientation for the images creates cumulative error on the end of the sequence. It should be consolidate with the control points or loop close imaging. The loop close imaging minimizes cumulative registration error that has been occurred sequential registration of the images. On the other hand, the control points both make registration of the point cloud model to extensive georeference reference frame and prevent from model deformations resulting from sequential registration of the images.

In this study, the object, which has $37 \mathrm{x} 47 \mathrm{~m}^{2}$ dimensions, was modelled by creating dense point cloud method, and reprojection error and georeferencing accuracy were evaluated for different imaging and control point configurations.

\section{DATA ACQUISITION}

The object images were recorded by Nikon P50 CCD camera that has $3264 \times 2448$ pixel array, $4.523 \mathrm{~mm}$ focal length and $5.46 \times 4.10 \mathrm{~mm}$ sensor dimensions. The imaging distances are about 15 meters. The eight traverse points were located around the structure for measuring ground based coordinates of the control points $(\mathrm{CPs})$ that were located on the object. Total 28 control points were created on the object and nine of them were selected as check point (ChP) (Fig. 1). CPs were signed with special target shape of photoscan software on paper. The coordinates of traverse and CPs were measured with Topcon OS-103 totalstation that has ability the measurement without reflector. The OS-103 totalstation has $3 \mathrm{~mm}+2 \mathrm{ppm}$ measurement accuracy on reflectorless mode (url-1).

* Corresponding author 


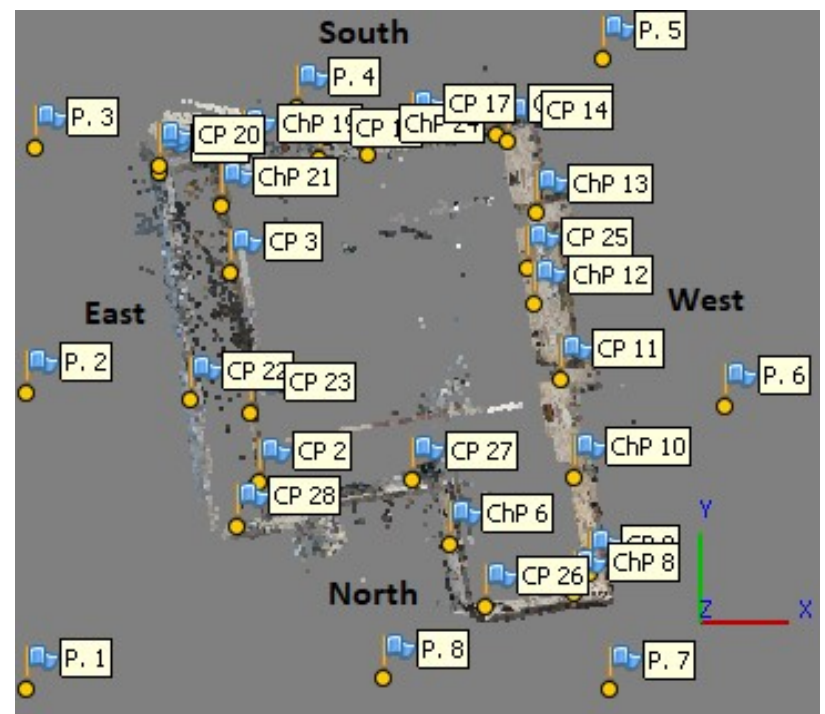

Fig. 1. Measurement object. (CP: Control point, ChP: Check point, P.: Traverse point)

\section{RESULTS}

\subsection{Dense Matching}

The keypoints from unordered images were created by using Photoscan software (Agisoft, 2017). The every keypoints were matched with the other images by similarities, and relative image positions and orientations were estimated. These procedures are called as structure from motion (SfM) in computer vision photogrammetry. The SfM based image matching results were given in Table 1 .

Table 1. Dense matching results

\begin{tabular}{|l|l|}
\hline Number of images & 46 \\
\hline Imaging distance & $15 \mathrm{~m}$ \\
\hline Ground resolution & $5.3 \mathrm{~mm} /$ pix \\
\hline Coverage area & $265 \mathrm{~m}^{2}$ \\
\hline Tie points \# & 62452 \\
\hline Projections \# & 150229 \\
\hline Reprojection error & $0.456 \mathrm{pix}$ \\
\hline Max reprojection error & $11.65 \mathrm{pix}$ \\
\hline
\end{tabular}

\subsection{Georeferencing}

The point cloud data were registered into georeference system by using control points. The registration has been performed with loop and without loop close imaging and varying control point configurations (Fig. 2). The set of the images have sequential overlapping regions around the structure. In the first configuration, the last image of the sequence was conjugated with the first of the order, and this imaging was called as loop close. In the second configuration, first and last images of the sequence does not have overlap region and conjugated points, this imaging was called as without loop close.

All of the CPs and ChPs have been identified from set of the images automatically in photoscan evaluation. The georeferencing was performed by means the CPs, and registration accuracy was evaluated with the residuals on the ChPs. The same check points were used in all tests for correct analysis of the registration accuracy. The residuals were computed by differences of coordinates that were measured and estimated (Eq. 1, Eq. 2, Eq. 3).
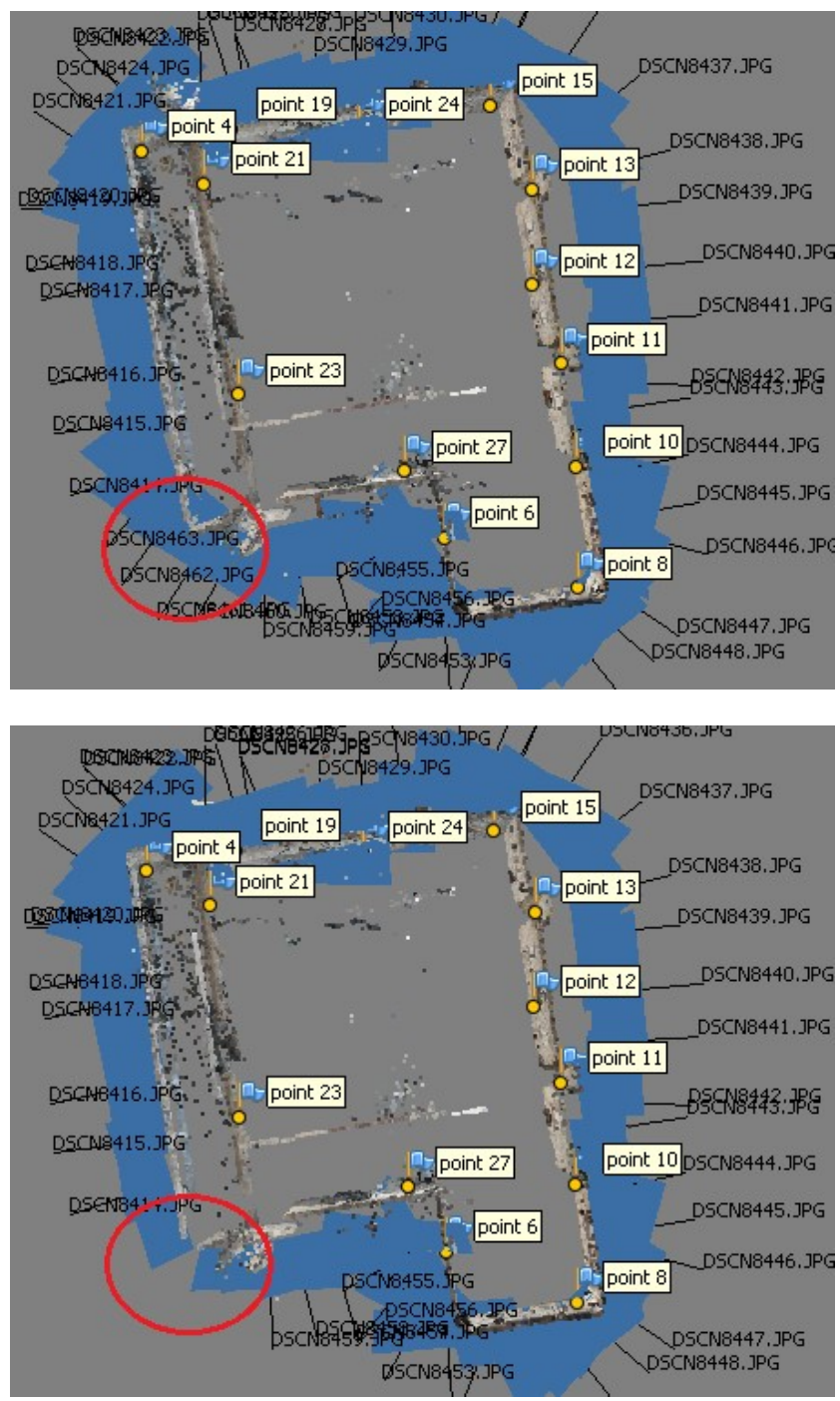

Fig. 2. Loop close (top) and without loop close (bottom) imaging.

$$
\begin{aligned}
& d_{z}=X_{m}-X_{e} \\
& d_{y}=Y_{m}-Y_{e} \\
& d_{z}=Z_{m}-Z_{e}
\end{aligned}
$$

Where, the subscripts $m$ and $e$ represent measured and estimated coordinates respectively. The overall accuracy of the registration was evaluated by the root mean square error (RMSE) of the coordinate residuals and the spatial errors on the XY plane and the XYZ coordinate space. The RMSE of the residuals were computed with below Equations (Eq. 4, Eq. 5, Eq. 6)

$$
\begin{aligned}
& R M S E_{d_{x}}=\sqrt{\frac{\sum\left(d_{x}\right)^{2}}{n}} ; R M S E_{d_{y}}=\sqrt{\frac{\sum\left(d_{y}\right)^{2}}{n}} ; \\
& \text { RMSE }_{d_{z}}=\sqrt{\frac{\sum\left(d_{z}\right)^{2}}{n}}
\end{aligned}
$$




$$
\begin{aligned}
& \operatorname{RMSE}_{d_{x y}}=\sqrt{\frac{\sum\left(d_{x}\right)^{2}+\Sigma\left(d_{y}\right)^{2}}{n}} \\
& \operatorname{RMSE}_{d_{x y z}}=\sqrt{\frac{\sum\left(d_{z}\right)^{2}+\Sigma\left(d_{y y}\right)^{2}+\Sigma\left(d_{z}\right)^{2}}{n}}
\end{aligned}
$$

Where $n$ is the number of points, which is nine for the ChPs. $d_{x y}, d_{x y z}$ are spatial errors in 2D space and 3D space respectively.

The first image configuration has loop close imaging, and the estimated residuals on the ChPs were given on Fig. 3 and Table 2. The second image configuration is on the without loop close imaging, and the estimated residuals on the ChPs were given on Fig. 4 and Table 3.
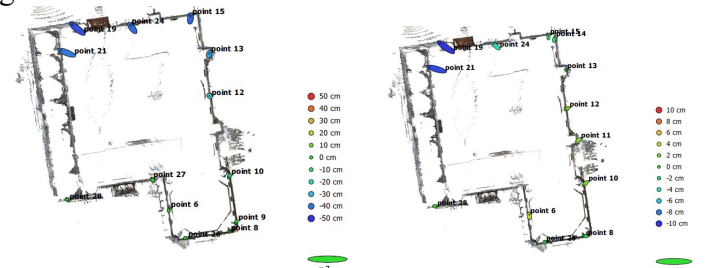

$4 \mathrm{CPs}$ on one facade (W) 4 CPs on two facades (W+S)
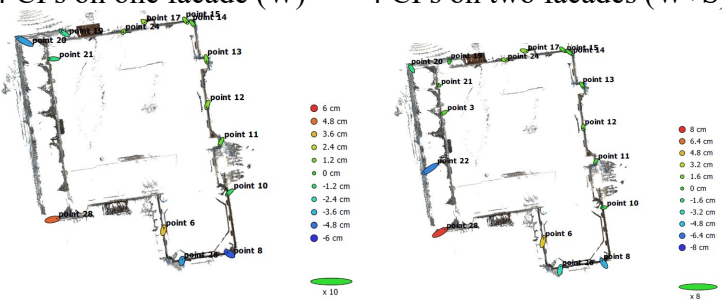

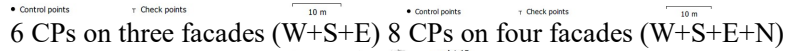
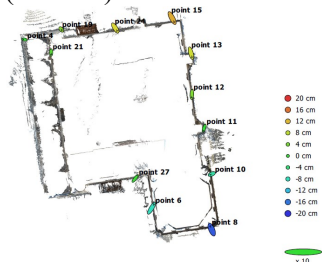

Uniform three CPs on all facades

Fig. 3. The residuals on CPs and ChPs in wihout loop close imaging (W:West, S: South, E: East, N: North)

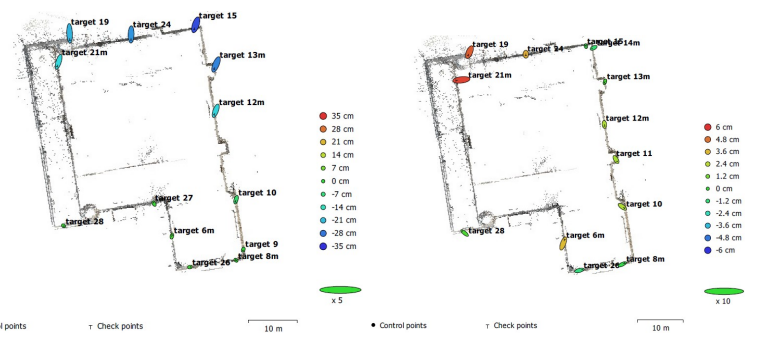

4 CPs on one facade (W) 4 CPs on two facades $(\mathrm{W}+\mathrm{S})$
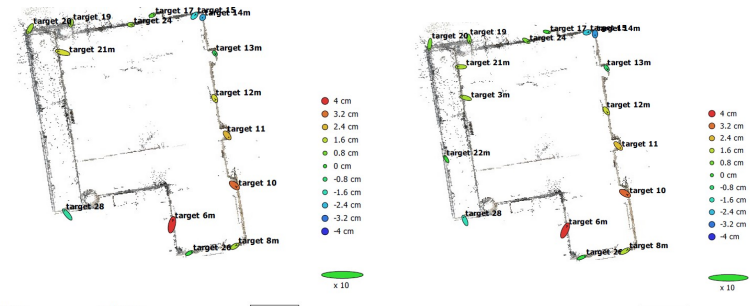

$6 \mathrm{CPs}$ on three facades $(\mathrm{W}+\mathrm{S}+\mathrm{E}) 8 \mathrm{CPs}$ on four facades $(\mathrm{W}+\mathrm{S}+\mathrm{E}+\mathrm{N})$

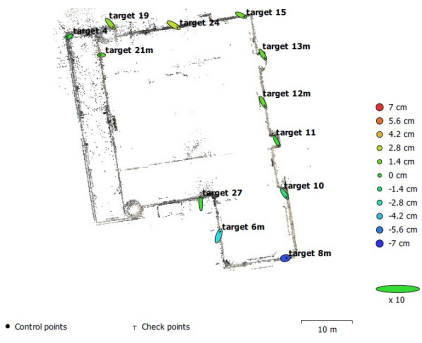

Uniform three CPs on all facades

Fig. 4. The residuals on CPs and ChPs in loop close imaging (W:West, S: South, E: East, N: North)

\begin{tabular}{|c|c|c|c|c|c|c|c|c|}
\hline Location & & $\begin{array}{l}\text { Count } \\
\# \\
\end{array}$ & Points name & $\begin{array}{l}\text { RMSE } \\
\mathrm{d}_{\mathrm{x}}\end{array}$ & $\begin{array}{l}\text { RMSE } \\
\mathbf{d}_{\mathbf{v}} \\
\end{array}$ & $\begin{array}{l}\text { RMSE } \\
d_{z} \\
\end{array}$ & $\begin{array}{l}\text { RMSE } \\
d_{x v} \\
\end{array}$ & $\begin{array}{l}\text { RMSE } \\
\mathbf{d}_{\mathrm{xvz}} \\
\end{array}$ \\
\hline 1 facade $(W)$ & $\mathrm{CP}$ & 4 & $9,26,27,28$ & 4.31 & 5.73 & 0.57 & 7.17 & 7.19 \\
\hline All facade & $\mathrm{ChP}$ & 9 & $6,8,10,12,13,15,19,21,24$ & 9.40 & 35.74 & 20.00 & 36.95 & 42.02 \\
\hline $\begin{array}{l}2 \text { facade } \\
(\mathrm{W}+\mathrm{S})\end{array}$ & $\mathrm{CP}$ & 4 & $11,14,26,28$ & 9.00 & 6.50 & 1.34 & 11.11 & 11.19 \\
\hline All facade & $\mathrm{ChP}$ & 9 & $6,8,10,12,13,15,19,21,24$ & 9.83 & 9.16 & 3.29 & 13.44 & 13.83 \\
\hline $\begin{array}{l}3 \text { facade } \\
(\mathrm{W}+\mathrm{S}+\mathrm{E})\end{array}$ & $\mathrm{CP}$ & 6 & $11,14,17,20,26.28$ & 7.87 & 8.99 & 1.67 & 11.90 & 12.02 \\
\hline All facade & ChP & 9 & $6,8,10,12,13,15,19,21,24$ & 8.50 & 8.49 & 2.20 & 12.01 & 12.21 \\
\hline $\begin{array}{l}4 \text { facade } \\
(\mathrm{W}+\mathrm{S}+\mathrm{E}+\mathrm{N})\end{array}$ & $\mathrm{CP}$ & 8 & $3,11,14,17,20,22,26,28$ & 9.01 & 9.87 & 1.52 & 13.36 & 13.45 \\
\hline All facade & ChP & 9 & $6,8,10,12,13,15,19,21,24$ & 9.23 & 9.44 & 2.14 & 13.20 & 13.38 \\
\hline $\begin{array}{l}\text { All facade } \\
\text { uniform }\end{array}$ & $\mathrm{CP}$ & 3 & $4,11,27$ & 6.57 & 16.19 & 0.13 & 17.47 & 17.47 \\
\hline All facade & $\mathrm{ChP}$ & 10 & $6,8,10,12,13,15,19,21,24$ & 10.31 & 11.98 & 2.83 & 15.81 & 16.06 \\
\hline
\end{tabular}

Table 2. With loop close, RMSE of residuals on CPs and ChPs [cm] 
Table 3. Without loop close, RMSE of residuals on CPs and ChPs [cm]

\begin{tabular}{|c|c|c|c|c|c|c|c|c|}
\hline Location & & $\begin{array}{l}\text { Count } \\
\#\end{array}$ & Points name & $\begin{array}{l}\text { RMSE } \\
d_{x}\end{array}$ & $\begin{array}{l}\text { RMSE } \\
d_{v}\end{array}$ & $\begin{array}{l}\text { RMSE } \\
d_{z}\end{array}$ & $\begin{array}{l}\text { RMSE } \\
d_{x v}\end{array}$ & $\begin{array}{l}\text { RMSE } \\
d_{\mathrm{xvz}}\end{array}$ \\
\hline 1 facade $(W)$ & $\mathrm{CP}$ & 4 & $9,26,27,28$ & 3.78 & 4.58 & 0.65 & 5.93 & 5.97 \\
\hline All facade & $\mathrm{ChP}$ & 9 & $6,8,10,12,13,15,19,21,24$ & 17.74 & 13.92 & 31.78 & 22.55 & 38.96 \\
\hline $\begin{array}{l}2 \text { facade } \\
(\mathrm{W}+\mathrm{S})\end{array}$ & $\mathrm{CP}$ & 4 & $11,14,26,28$ & 4.07 & 5.11 & 1.82 & 6.53 & 6.78 \\
\hline All facade & $\mathrm{ChP}$ & 9 & $6,8,10,12,13,15,19,21,24$ & 16.23 & 9.38 & 5.08 & 18.75 & 19.42 \\
\hline $\begin{array}{l}3 \text { facade } \\
(W+S+E)\end{array}$ & $\mathrm{CP}$ & 6 & $11,14,17,20,26.28$ & 13.13 & 8.84 & 3.30 & 15.83 & 16.17 \\
\hline All facade & $\mathrm{ChP}$ & 9 & $6,8,10,12,13,15,19,21,24$ & 9.02 & 8.32 & 2.41 & 12.27 & 12.50 \\
\hline $\begin{array}{l}4 \text { facade } \\
(\mathrm{W}+\mathrm{S}+\mathrm{E}+\mathrm{N})\end{array}$ & $\mathrm{CP}$ & 8 & $3,11,14,17,20,22,26,28$ & 16.93 & 13.95 & 3.80 & 21.94 & 22.27 \\
\hline All facade & $\mathrm{ChP}$ & 9 & $6,8,10,12,13,15,19,21,24$ & 7.51 & 11.14 & 2.44 & 13.44 & 13.66 \\
\hline $\begin{array}{l}\text { All facade } \\
\text { uniform }\end{array}$ & $\mathrm{CP}$ & 3 & $4,11,27$ & 6.77 & 9.76 & 0.17 & 11.87 & 11.87 \\
\hline All facade & $\mathrm{ChP}$ & 10 & $6,8,10,12,13,15,19,21,24$ & 7.00 & 14.41 & 9.66 & 16.02 & 18.70 \\
\hline
\end{tabular}

\subsection{Reprojection error}

The reprojection error is an error corresponding to the image distance between a measured and projected point. The back projection of the point onto the image has been made by estimated orientation and location parameters of the image. The size of the reprojection error is related with the measured image coordinates, intersection angle between point of view line of overlapping images and base/height ratio. Dense image matching usually has got a lot of images. Base/height ratio and an intersection angle of point of view lines does not make any negative effect to the reprojection accuracy. However, the accuracy of measured image point location is direct related with the accuracy of reprojection error. The point position on the image is detected automatically in dense matching. Thus image point measurement is only related keypoint localization, characteristics of imaging area and perspective distortion. The perspective distortion defines the point of view angle with respect to the imaged surface. In this study, for varying point of view angle, in other words for different base/height ration, the reprojection error was estimated (Fig. 5). Root mean square (RMS) of the residuals was computed below equations (Remondino et al., 2017)

$$
\begin{aligned}
& \text { RMS }_{x}=\sqrt{\frac{1}{n} \cdot \sum_{i=1}^{n}\left(x_{i}-\overline{x_{i}}\right)^{2}} \\
& R M S_{y}=\sqrt{\frac{1}{n} \cdot \sum_{i=1}^{n}\left(y_{i}-\overline{y_{i}}\right)^{2}} \\
& R M S=\sqrt{R M S_{x}{ }^{2}+R^{2} M S_{y}{ }^{2}}
\end{aligned}
$$

Where $\left(x_{i}, y_{i}\right)$ represent the image coordinates, i.e. the position of the matched $2 \mathrm{D}$ points, and $\left(\overline{x_{i}}, \overline{y_{i}}\right)$ are the reprojected values of the object point coordinates computed within the bundle adjustment procedure. The reprojection error was computed for different base/height ratio of stereo images (Table 4).

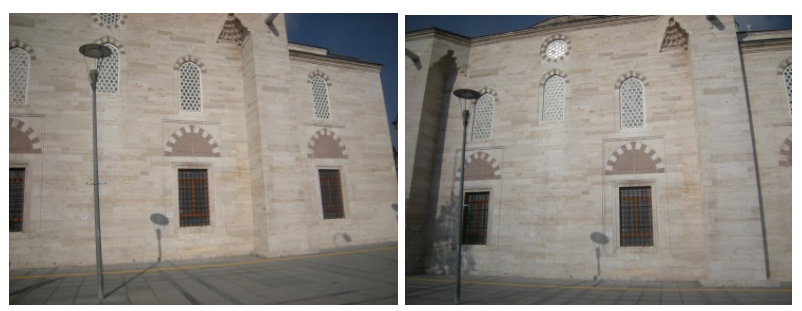

$\mathrm{b} / \mathrm{h}=0.34$
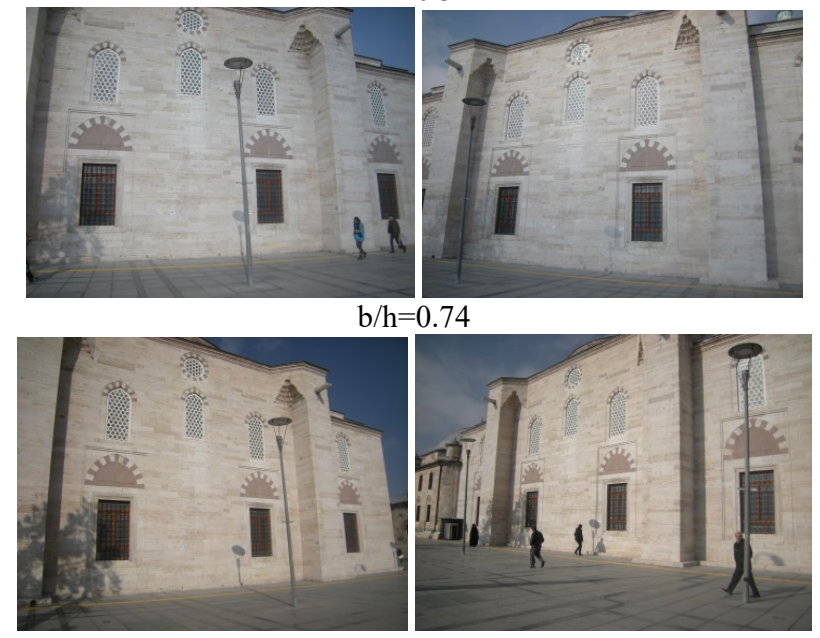

$\mathrm{b} / \mathrm{h}=1.56$

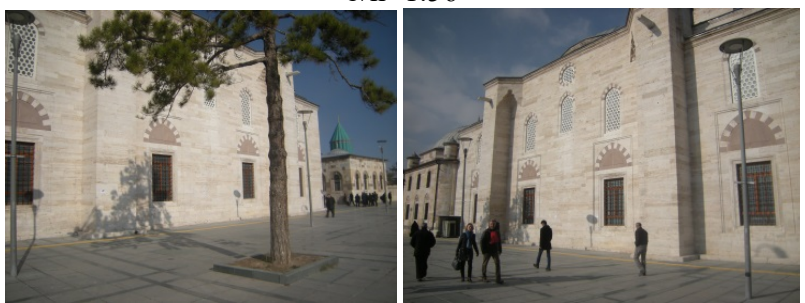

$\mathrm{b} / \mathrm{h}=2.16$

Fig. 5. The images that have different base length ratio

Table 4. Reprojection error for different base/height ratio.

\begin{tabular}{|c|c|c|c|c|}
\hline $\begin{array}{c}\text { Height } \\
{[\mathrm{m}]}\end{array}$ & $\begin{array}{c}\text { Base } \\
{[\mathrm{m}]}\end{array}$ & $\mathrm{b} / \mathrm{h}$ & $\begin{array}{c}\text { RMS } \\
\text { projection } \\
\text { error [pixel] }\end{array}$ & $\begin{array}{c}\text { Max. } \\
\text { reprojection } \\
\text { error [pixel] }\end{array}$ \\
\hline 14.60 & 4.94 & 0.34 & 0.99 & 3.97 \\
\hline 14.60 & 10.74 & 0.74 & 0.38 & 2.58 \\
\hline 14.60 & 22.77 & 1.56 & 0.59 & 1.53 \\
\hline 14.60 & 31.59 & 2.16 & 0.24 & 1.18 \\
\hline
\end{tabular}




\section{DISCUSSION}

The results show that the sequential registration of the images creates cumulative error. The registration of open loop imaging with 4 CPs was caused high residuals on the end close images (Table 2). The control points reduce the cumulative registration errors in the registration of the model into geodetic system. The distribution of CPs is more important than their number. It should be uniform or not clustered in a small region. The georeferencing that was performed with $3 \mathrm{CPs}$ has similar residuals on the ChPs with the registration of more CPs. The loop close imaging restricts cumulative error of image orientation. Nevertheless their georeferencing should be performed with uniform distributed CPs (Table 3).

The size of reprojection error is generally small in multiview registration opposite to stereo images. The results were shown that the small base length has more conjugate keypoints that have large reprojection errors. The reprojection error was reduced with the long base length. The relative orientation has been executed by least five conjugate points. However automatically extracted keypoints enables more conjugate points in overlapping area, and more conjugate keypoints than ten does not make improvement the orientation accuracy (Luhmann et al., 2007; Fraser, C.S., 2005). Besides, the photogrammetry requires proper base/height ratio for high accuracy evaluation. In this study RME of reprojection error was 0.99 pixel and max reprojection error was 3.97 pixel for 0.34 base height ratio. They were acquired as 0.24 and 1.18 pixel respectively for 2.16 base height ratio .

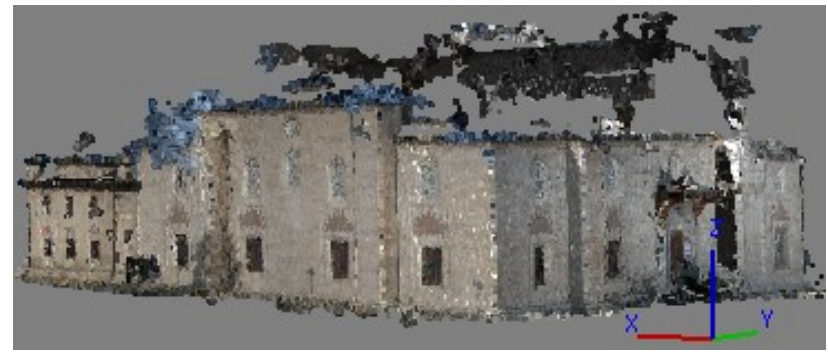

Fig.6. Point cloud model

\section{CONCLUSION}

Dense point cloud method is low cost and easy method for 3D digitization of historical structures. Consecutive registration of a large number of the images generates cumulative orientation error. Thus it must be keep down via loop close imaging. On the other hand ground control points hold in low level to registration error while registering to model into geodetic system. The control points have to be uniform distribution at least three numbers. The corridor mapping such as transmission line, road and long narrow areas also includes sequential registration of the images. In this situation, model accuracy must be assured by the control points which have been located as uniform distribution in all over the measurement area.

\section{REFERENCES}

Agisoft, 2017. Photoscan Professional (Ver.:1.3.2.4205).

Barazzetti, L., Remondino, F., Scaioni, M., 2011. Automated and accurate orientation of complex image sequences. International Archives of the Photogrammetry, Remote Sensing and Spatial Information Sciences, Vol. XXXVIII-5/W16, pp. 277-284.

Fraser, C.S., 2005. Network orientation models for image-based 3D measurement, International Archives of Photogrammetry, Remote Sensing and Spatial Information Sciences, 36(5/W17).

Luhmann, T., Robson, S., Kyle, S., Harley, I. 2007, Close Range Photogrammetry: Principles. Techniques and Applications, Wiley Whittles, Caithness, pp. 116-227.

Piermattei, L., 2016. The use of structure from motion technologies for high-resolution terrain modelling on high altitude cathchments. PhD Thesis, University of Padova, Department of Land, Environment, Agriculture and Forestry (TESAF), Italy.

Remondino, F., Nocerino, E., Toschi, I., Menna, F., 2017. A critical review of automated photogrammetric processing of large datasets. The International Archives of the Photogrammetry, Remote Sensing and Spatial Information Sciences, Vol. XLII-2/W5, pp. 591-599.

Verhoeven, G., Karel, W., Štuhec, S., Doneus, M., Trinks, I., Pfeifer, N. 2015. Mind your grey tones - examining the influence of decolourization methods on interest point extraction and matching for architectural image-based modelling. 3D-Arch 2015, 25-27 February, Avila, Spain. The International Archives of the Photogrammetry, Remote Sensing and Spatial Information Sciences, Vol. XL-5/W4, pp.307-314.

Wilkinson, M.W., Jones, R.R., Woods, C.E., Gilment, S.R., McCaffrey, K.J.W., Kokkalas, S., Long, J.J., 2016. A comparison of terrestrial laser scanning and structure-frommotion photogrammetry as methods for digital outcrop acquisition: Geosphere 12(6): 1865-1880, doi: 10 $.1130 / \mathrm{GES} 01342.1$.

url-1: https://www.topcon.co.jp/en/positioning/products/pdf/OS E.pdf [Acces: 15 March, 2019] 